INGENIERÍA SANITARIA Y AMBIENTAL

\title{
Sostenibilidad financiera y económica de plantas de manejo de residuos sólidos urbanos en Colombia
}

INGENIERÍA SANITARIA Y AMBIENTAL

\section{Financial and economic sustainability of urban solid waste recovery facilities in Colombia}

\author{
Carlos A. Ramírez-Vargas*§, Diego Paredes*, Jhoniers Guerrero* \\ *Grupo de Investigación en Agua y Saneamiento, Universidad Tecnológica de Pereira, Pereira, Colombia \\ §c.a.ramirez@utp.edu.co,diparede@utp.edu.co,jhguerre@utp.edu.co
}

\begin{abstract}
Resumen
En Colombia menos del 2\% de los Residuos Sólidos Urbanos Domiciliarios (RSUD) son manejados en Plantas de Manejo de Residuos Sólidos (PMRS), presentando en la mayoría de casos perdidas operacionales cercanas al 60\%, llevando al cierre de gran parte de dichas instalaciones. Ante ello, esta investigación se enfocó en establecer unas condiciones mínimas para un escenario económico-financiero de operación sostenible de PMRS en municipios menores a 50,000 habitantes, mediante la combinación de herramientas de Dinámica de Sistemas y Análisis Estructural. Como resultado se identificó que i) la cantidad de material potencialmente aprovechable a recibir, ii) los ingresos por tarifa de recepción de material y iii) las cantidades de material recuperado para la venta, se constituyen como Variables Clave de sostenibilidad. Para el escenario simulado se concluye que con el actual cobro de tarifas de recepción de residuos, el aumento de eficiencias de recuperación y comercialización de residuos, sería necesario la recepción de $302 \mathrm{t} / \mathrm{mes}$ para alcanzar una relación beneficio/costo igual a 0 . Sin embargo dicho valor puede variar en función de las particularidades regionales y tecnologías implementadas de cada proyecto PMRS, lo cual hace necesario el desarrollo de estudios puntuales para el desarrollo de un proyecto en una localidad determinada.
\end{abstract}

Palabras clave: Diagrama causal, dinámica de sistemas, gestión integrada de residuos sólidos, MICMAC, variables clave.

\begin{abstract}
In Colombia, less that $2 \%$ of Urban Solid Waste (USW) are treated in Solid Waste Recovery Facilities (SWRF), having in almost all cases operational losses close to $60 \%$, leading to the closure of several plants. Based on that, this research was focused on the establishment of some minimal conditions for the sustainability of a financialeconomic scenario of a SWRF in towns with less than 50,000 inhabitants, by combining System Dynamics and Structural Analysis tools. As result, it was identified that the i.) amount of potentially usable material received, ii.) incomes for material reception, and iii.) amount of recovered material for sale, become as Key of sustainability. For the simulated scenario it is concluded that with the current fee of solid waste reception, the increase of recovery efficiency and merchandising of materials, it would be necessary to receive at least $302 \mathrm{t} / \mathrm{month}$, to reach a cost/ benefit ratio equal to 0 . However that value can change as a function of the regional characteristics and implemented technologies of each project, thus it is necessary the development of studies of a project in a determined location.
\end{abstract}

Keywords: Causal diagram, integrated solid waste management, key variables, MICMAC, system dynamics. 


\section{Introducción}

La disposición de residuos sólidos es un problema en constante crecimiento Jha et al (2011) y puede causar daños a la salud humana y el ambiente (Vergara \& Tchobanoglous, 2012). Es un tema de preocupación mundial debido al incremento poblacional y a los cambios de patrones de consumo (Marshall \& Farahbakhsh, 2013). A nivel global en el 2001 se generaron 680 millones de toneladas de residuos sólidos en áreas urbanas (0.64 kg/hab/d), para el 2011 aumentó a 1300 millones $(1.2 \mathrm{~kg} / \mathrm{hab} / \mathrm{d})$ y se estima que al 2025 se generarán 2200 millones $(1.42 \mathrm{~kg} / \mathrm{hab} / \mathrm{d})$ (Hoorweg \& Bhada-Tata, 2012).

El manejo de residuos sólidos ha trascendido de esquemas de disposición final en relleno sanitario hacia sistemas integrados de gestión, donde los residuos se consideran como un recurso aprovechable (Dong et al., 2014; Laurent et al., 2014), que aparte de contemplar aspectos técnicos, también deben garantizar la sostenibilidad de tales sistemas a lo largo del tiempo (Zurbrügg et al., 2012).

En paises en vías de desarrollo el manejo de residuos sólidos presenta dificultades, debido a volúmenes de generación creciente, altos costos de gestión y limitaciones de conocimiento respecto a los factores que influyen en su manejo adecuado (Abarca et al., 2013). En América Latina y el Caribe los esfuerzos de las autoridades locales para el fortalecimiento de los sistemas de gestión de residuos sólidos son limitados por los hábitos de consumo, la creciente tasa de generación, composición, coberturas de recolección y prácticas de manejo inadecuadas, sumado a dificultes para lograr de finanzas sostenibles y establecimiento de cooperación público-privada (BID et al., 2011; PNUMA, 2013). Si bien la gestión de residuos sólidos despierta el interés de muchos actores sociales, en América del Sur la principal práctica sigue siendo la disposición final sin ningún tipo de tratamiento, generando impactos ambientales (Bezama et al., 2007).

Las Plantas de Manejo de Residuos Sólidos (PMRS) son unidades productivas orientadas al aprovechamiento y valorización de residuos sólidos con el fin de obtener productos con estándares de calidad que permitan reincorporarlos en otros procesos productivos (Marmolejo et al., 2009; Oviedo-Ocaña et al., 2011,). En Colombia, las PMRS han tenido acogida por parte de numerosos municipios menores en el país (Marmolejo et al., 2011; Victoria et al., 2012). Sin embargo existen inquietudes relacionadas con las cantidades de residuos sólidos manejados en dichas plantas, lo cual se refleja en drásticas reducciones en la cantidad de residuos gestionados al año, pasando de 615 t a 212 t entre 2008 y 2012 (Superintendencia de Servicios Públicos Domiciliarios, 2009 y 2013).

Lo anterior se atribuye a que la normatividad asociada a la operación de PMRS es dispersa y desactualizada, el aprovechamiento se realiza como medida de choque frente al fin de la vida útil de los rellenos sanitarios y no como proyectos planificados, se ha omitido la consideración del manejo de residuos sólidos como una actividad industrial, y mucho menos se han consolidado las cadenas de comercialización de materiales recuperados que garanticen precios justos (CONPES, 2008; SSPD, 2008).

Estas circunstancias en combinación con bajos ingresos por cobro de recepción de materiales en las PMRS, han conducido a reportes financieros comprometedores, con déficits de costos del 35\% y pérdidas operacionales cercanas al $60 \%$ (SSPD, 2008), generado un panorama de sostenibilidad económico-financiero comprometedor para las plantas instaladas en el país. Dicha situación reviste especial interés en municipios con poblaciones inferiores a 50,000 habitantes, ya que presentan dificultades de índole técnico, administrativo, económico y socio-cultural que afectan negativamente la gestión integral de residuos sólidos (UNICEF et al, 2005). Considerando tal panorama, la investigación se enfocó en establecer unas condiciones mínimas para alcanzar la sostenibilidad financiera $y$ económica de las PMRS en Colombia, mediante el uso de herramientas de dinámica de sistemas y análisis estructural. 


\section{Metodología}

\subsection{Localización}

Se seleccionaron y tomaron registros provenientes de 28 PMRS (Tabla 1), reportadas en el estudio sectorial elaborado por la Superintendencia de Servicios Públicos Domiciliarios de Colombia (SSPD, 2008), para el cual la fuente recolectó información en visitas a los sitios seleccionados y mediante solicitudes de información a autoridades ambientales y personal administrativo de dichas PMRS. La investigación se desarrolló en 4 etapas comprendidas por: i.) la identificación de variables genéricas de funcionamiento de PMRS, ii.) el desarrollo de un diagrama causal, iii.) la identificación de variables clave y, iv) la estimación de costos y beneficios de PMRS tipo.

\subsection{Variables asociadas al funcionamiento de PMRS}

En la investigación se revisaron documentos relacionadas con aspectos técnicos, sociales, normativos, políticos, económicos y ambientales, que tienen injerencia en el desarrollo de actividades de aprovechamiento en el país. A partir de ello se definió un conjunto de treintaitrés (33) variables (Tabla 2), asociadas con las diferentes etapas de un esquema de servicio de aseo urbano tipo. Las variables fueron categorizadas como Cuantificables (operativas, económicas y financieras) y de Soporte (normativas, políticas y sociales), para facilitar la representación mediante un diagrama causal del sistema y para posteriores análisis de las estructuras de costos de las PMRS.

\subsection{Desarrollo de diagrama causal}

La dinámica relacionada con la operación de las PMRS en el país, se representó mediante la elaboración de un Diagrama Causal, con base en. Forrester (Toole, 2005; Torres \& Olaya, 2010). Estos diagramas se componen de variables conectadas que denotan influencias causales entre sí, mediante la asignación de polaridad positiva (+) o negativa (-) dependiendo del tipo de cambio de la variable influenciada. Una relación positiva implica que un aumento en la variable "A" provoca un aumento en la variable "B" (y viceversa), mientras que una relación negativa denota que un aumento de la variable " $A$ " conduce a la disminución de la variable "B" (y viceversa). El diagrama elaborado posibilitó identificar la dinámica entorno al sistema estudiado y explicar los efectos de las variables involucradas.

\subsection{Identificación de variables clave}

Cada variable del diagrama causal fue analizada en términos del grado de influencia y dependencia respecto a las demás variables, para identificar aquellas que por su alto valor de influencia, dependencia y acciones indirectas puedan ser consideradas como Variables Clave. Para ello, el análisis se desarrolló con la ayuda de la Matriz de Influencias Directas del software MICMAC (Laboratorio de Investigación Prospectiva $\mathrm{y}$ Estratégica - LIPSOR, París). Con dicha matriz se efectuó la valoración directa del conjunto de variables analizadas, mediante la calificación manual del grado de influencia de una variable sobre las demás, acorde con las interrelaciones establecidas en el diagrama causal y considerando los parámetros de calificación establecidos en la Tabla 3. La matriz alcanzó una tasa de valoración (calificaciones diferentes a cero) del $25.25 \%$, porcentaje inferior al $30 \%$ que es el máximo recomendado por Godet et al. (2007), garantizando la validez de la calificación. A partir de la sumatoria de las calificaciones de las filas y las columnas de la matriz se obtuvo el grado de influencia y dependencia de cada variable.

A partir de la valoración directa, se efectuó la valoración indirecta mediante el uso de la Matriz de Impactos Cruzados. Esta valoración fue generada mediante la programación del software MICMAC, para la ejecución de multiplicaciones de la matriz de valoración directa para estabilizar el sistema. Acorde a Cano (2011) la estabilización del sistema se da a partir de la potencia en la cual el orden jerárquico de las variables (en función del grado de motricidad y dependencia) se mantiene, lo cual para el caso de las variables analizadas se dio a partir de cinco (5) multiplicaciones. Con los 


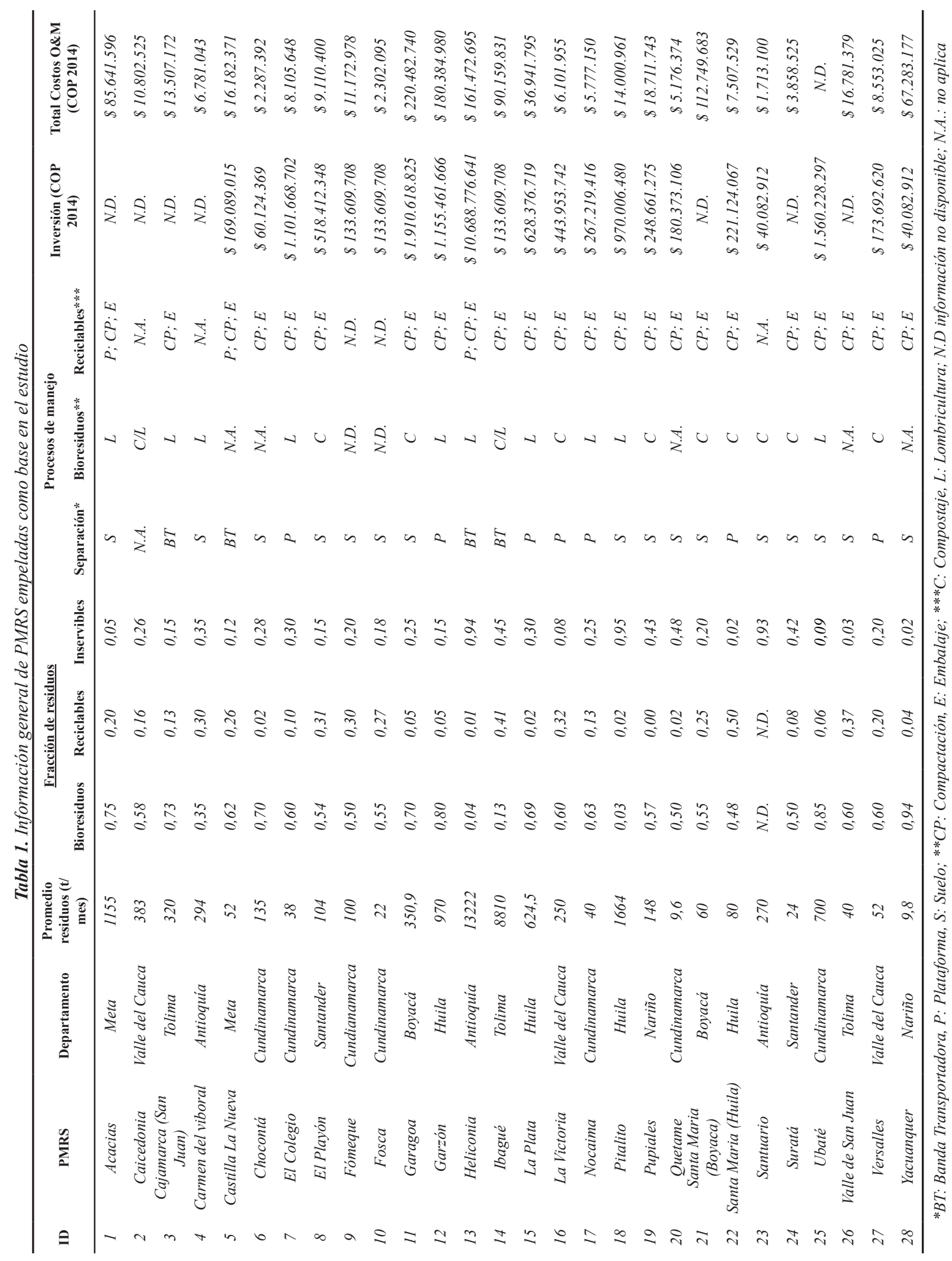


Tabla 2. Variables de análisis dinámica de PMRS en Colombia

\begin{tabular}{|c|c|c|c|c|}
\hline$N^{\circ}$ & Título largo & Título corto & Componente* & Tipo** \\
\hline 1 & Economía de escala por concepto de regionalización & $\$ E S c$ & $R \& T$ & $S$ \\
\hline 2 & Peso de residuos generados & $W R S$ & $R \& T$ & $C$ \\
\hline 3 & Peso de residuos separados en la fuente & $W R S$ fuent & $R \& T$ & $C$ \\
\hline 4 & Peso de residuos minimizados en la fuente & $W R S \min$ & $R \& T$ & $C$ \\
\hline 5 & $\begin{array}{c}\text { Implementación de PGIRS por parte de autoridades locales (R\&Ty } \\
\text { Aprovechamiento) }\end{array}$ & PGIRS & $R \& T$ & $S$ \\
\hline 6 & $\begin{array}{c}\text { Fomento de esquemas de regionales de aprovechamiento (transporte } \\
\text { hasta plantas regionales) }\end{array}$ & Foment Reg & $R \& T$ & $S$ \\
\hline 7 & Política GIRS 1997 & Pol GIRS/97 & $R \& T$ & $S$ \\
\hline 8 & Titulo F RAS 2000 & RAS 2000 & $R \& T$ & $S$ \\
\hline 9 & Decreto 1713 de 2002 & Dec1713/02 & $R \& T$ & $S$ \\
\hline 10 & $\begin{array}{c}\text { Resolución CRA } 351 \text { de 2005: Art 17. "Aprovechamiento" (Costos de } \\
\text { transporte por tonelada y tramo excedente) }\end{array}$ & Res $351 / 05$ & $R \& T$ & $S$ \\
\hline 11 & Pago por disposición final & $\$ D F$ & $A P R$ & $C$ \\
\hline 12 & Ingresos por tarifa de recepción de material a aprovechar & IN\$ tarifa & $A P R$ & $C$ \\
\hline 13 & Costos de Inversión & \$inver & $A P R$ & $C$ \\
\hline 14 & Costos de Operación & \$oper & $A P R$ & $C$ \\
\hline 15 & Gastos Generales & Gastos gen & $A P R$ & $C$ \\
\hline 16 & Peso de MPA a recibir & W MPA reci & $A P R$ & $C$ \\
\hline 17 & $\begin{array}{l}\text { Método de selección (suelo, plataforma - tolva, bandas transportadoras; } \\
\text { tecnificado, manual) }\end{array}$ & Met selecc & $A P R$ & $S$ \\
\hline 18 & Peso de MPA clasificado & W MPA cla & $A P R$ & $C$ \\
\hline 19 & Peso de Material Descartable generado & $W$ desc & $A P R$ & $C$ \\
\hline 20 & Eficiencia de recuperación & Efic recup & $A P R$ & $C$ \\
\hline 21 & $\begin{array}{l}\text { Tipo de Tratamiento (Tratamiento de Material Orgánico separado } \\
\text {-Compostaje, Lombricultivo; beneficio de material recuperable) }\end{array}$ & Tipo trat & $A P R$ & $S$ \\
\hline 22 & Capacidad instalada de aprovechamiento necesaria & Cap instal & $A P R$ & $C$ \\
\hline 23 & Normatividad Ambiental & Norma Amb & $A P R$ & $S$ \\
\hline 24 & $\begin{array}{l}\text { Beneficios ambientales en el sitio de disposición final (reducción de } \\
\text { generación de lixiviados, de emisiones de gases efecto invernadero, etc) }\end{array}$ & $Q a m b D F$ & $A P R$ & $S$ \\
\hline 25 & Calidad de MPA clasificado & $Q M P A$ cla & $A P R$ & $S$ \\
\hline 26 & Demanda de materiales recuperados (Peso) & Deman rec & $C O M$ & $C$ \\
\hline 27 & Ingresos por comercialización de material recuperado & IN\$ recup & $C O M$ & $C$ \\
\hline 28 & Otros ingresos (ej: Bonos CER; protocolo de Kioto) & IN\$ otros & $C O M$ & $C$ \\
\hline 29 & Comercialización de material con valor agregado & Valor agre & $C O M$ & $S$ \\
\hline 30 & RBC (Relación Beneficio - Costo) y BN (Beneficio Neto) & $R B C / B N$ & $C O M$ & $C$ \\
\hline 31 & Peso de material recuperado para la venta & W mat vent & $C O M$ & $C$ \\
\hline 32 & $\begin{array}{c}\text { Estímulos a empresas para efectos de incorporación de materias primas } \\
\text { recuperadas en los procesos de aprovechamiento }\end{array}$ & Estim comp & $C O M$ & $S$ \\
\hline 33 & $\begin{array}{c}\text { Res ICA } 00375 \text { de } 2004 \text { "Por la cual se dictan las disposiciones sobre } \\
\text { Registro y Control de los Bioinsumos y Extractos Vegetales de uso } \\
\text { agrícola en Colombia." }\end{array}$ & Res $375 / 04$ & $C O M$ & $S$ \\
\hline
\end{tabular}

*R\&T: Recolección y Transporte; APR: Aprovechamiento; COM: Comercialización. ** C: Cuantificable; S: Soporte 
Tabla 3. Calificación de relación entre variable evaluadas

\begin{tabular}{ccc}
\hline Grado de Influencia & Calificación* & Criterio de Calificación \\
\hline Potencial & $P(4)$ & $\begin{array}{c}\text { Relación de influencia potencial de variables de cuarto orden (mediada por } \\
\text { tres variables intermedias) o por variables de soporte no cuantificables cuya } \\
\text { influencia pueda manifestarse en un futuro cercano en caso tal de darse las } \\
\text { condiciones propicias para ello. }\end{array}$ \\
Fuerte & 3 & Relación de influencia de primer orden (directa) de una variable sobre otra. \\
Medio & 2 & Relación de influencia de segundo orden, mediada por una variable intermedia. \\
Débil & 1 & Relación de influencia de tercer orden, mediada por dos variables intermedias. \\
Nulo & 0 & No existe relación entre variables.
\end{tabular}

*Escala de calificación con base en valores predeterminados del software MICMAC.

resultados de la valoración indirecta, se generó el Plano de Motricidad/Dependencia Indirecta Potencial, el cual clasifica las variables en cuadrantes dependiendo de su grado de influencia y dependencia. Las variables de interés para esta investigación son aquellas con alto grado de influencia y dependencia (Variables Clave), dado que cualquier acción que se genere en ellas, tendrá un efecto cascada sobre el resto de variables.

\subsection{Estimación de costos y beneficios de PMRS tipo}

A partir de las variables clave identificadas e información financiera empleada por SSPD (2008), se compuso una estructura de costos y beneficios para una PMRS tipo con cifras actualizadas al año 2014 con base en los valores históricos del índice de precios al consumidor IPC. La estimación de costos de inversión y de operación y mantenimiento se efectuó con las Ec. (1) y Ec. (2).

$$
\begin{aligned}
& \text { Inv }=12.874 .283,53 x^{0.65} \quad R^{2}=0.86 \\
& \text { OyM=2.326.567.90x } x^{-0.74} \quad R^{2}=0.65
\end{aligned}
$$

Con la Ec. (1) se estimaron los costos de inversión (Inv; \$/t-mes) en función de la capacidad instalada de la PMRS ( $x$; t/mes), y con la Ec. (2) los costos de operación y mantenimiento (OyM; $\$ /$ t-mes). Los beneficios se estimaron a partir de la generación de ingresos por recepción de residuos y comercialización de materiales recuperados, con base en una serie de constantes asumidas (Tabla 4). Una vez identificados los costos y beneficios, se hizo su estimación anual para un periodo de 15 años, correspondiente a la vida útil de una PMRS. Posteriormente, fue calculado el Beneficio Neto (BN) y la Relación Beneficio-Costo (RBC) del Valor Presente (VP) al año 2014, mediante el uso de las Ec. (3), (4) y (5) (Field \& Field, 2012).

$$
\begin{gathered}
B N=B T-C T \\
V P=V F /(1+i)^{\mathrm{t}} \\
R B C=B T / C T
\end{gathered}
$$

En dichas ecuaciones "BT" se refiere al beneficio total, "CT" al costo total, "VF" al valor futuro, "i" a la tasa de descuento y " $\mathrm{t}$ " al año de proyección. Dado que de que no existe un consenso respecto a la tasa de descuento idónea para aplicar en una situación determinada, se seleccionó un valor "i" de $12 \%$, con base en datos reportados por la Comisión de Regulación de Agua Potable y Saneamiento (CRA, 2006).

\section{Resultados y discusión}

\subsection{Diagrama causal representativo del aprovechamiento de RSUD en Colombia}

A partir de las variables identificadas, se construyó un diagrama causal aplicable a la situación de sostenibilidad financiera-económica de las PMRS en Colombia (Figura 1; Tabla 2), en el cual es posible identificar tres (3) conglomerados de variables. El primer conglomerado se compone de variables asociadas con la cantidad de residuos generados y que tienen potencial de 


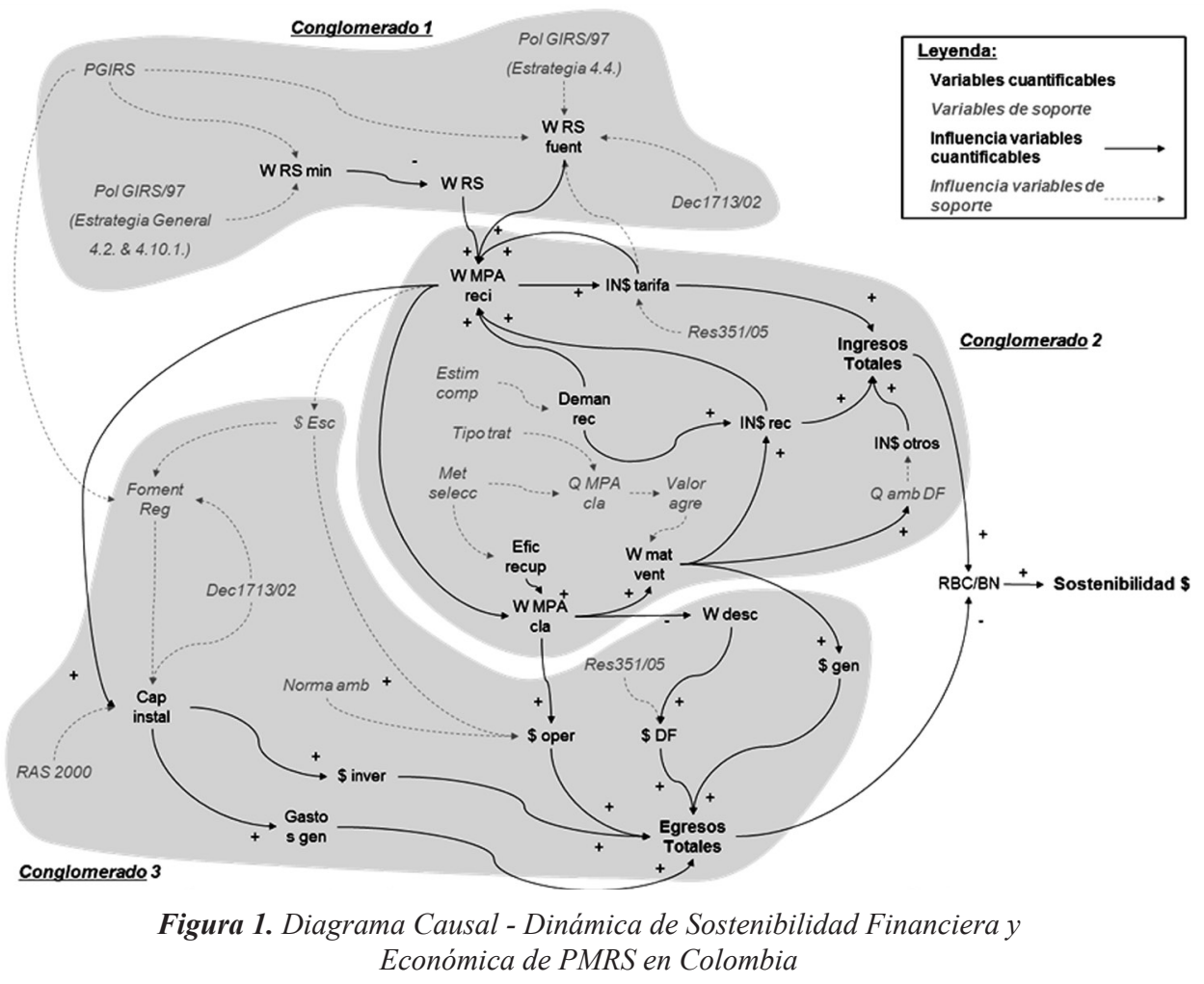

aprovechamiento en las PMRS. Allí se aprecia que las cantidades a recibir se ven afectadas por la existencia o no de prácticas de reducción y de separación en la fuente, que a su vez están condicionadas por la implementación de los respectivos planes de gestión integral de residuos sólidos (PGIRS) y de la normatividad vigente aplicable a la gestión de residuos sólidos por parte de las autoridades responsables de ello.

En el segundo conglomerado se agrupan una serie de variables que afectan la generación de ingresos en las PMRS. Entre ellas se destaca la cantidad de residuos a recibir, ya que condiciona los ingresos por concepto de tarifa de recepción de materiales potencialmente aprovechables y a su vez genera estímulos para la recepción de nuevos residuos. Por su parte la comercialización de materiales recuperados, afectan los ingresos ya que en la medida en estos se venden, generan ingresos y estimulan la recepción de nuevos residuos en las PMRS. A su vez la venta de materiales recuperados se ve influenciada por la demanda existente en los mercados regionales, al igual que por aspectos técnicos como los métodos de selección y tratamiento, que afectan la eficiencia y la calidad de los materiales recuperados.

En el tercer conglomerado se agrupan variables que influyen sobre los costos totales de una PMRS. Allí se destaca que el aumento de la capacidad instalada (supeditada a la disponibilidad de material potencialmente aprovechable a recibir), influencia el aumento en los costos de inversión y gastos generales, al igual que disminuyen los costos de operación por efecto de economía de escala. Igualmente en la medida en que los costos de operación se afectan por la eficiencia de los métodos de selección y tratamiento, los costos totales también lo hacen. Otra variable a destacar es la generación de material descartado, el cual depende de la eficiencia de recuperación de materiales de la planta, ya que entre menores cantidades se descarten, menores serán sus costos de disposición final y viceversa.

A partir de las interacciones de las variables en el diagrama es posible identificar su influencia tanto en los ingresos totales como de los costos totales, los cuales influencian el valor del beneficio neto y 


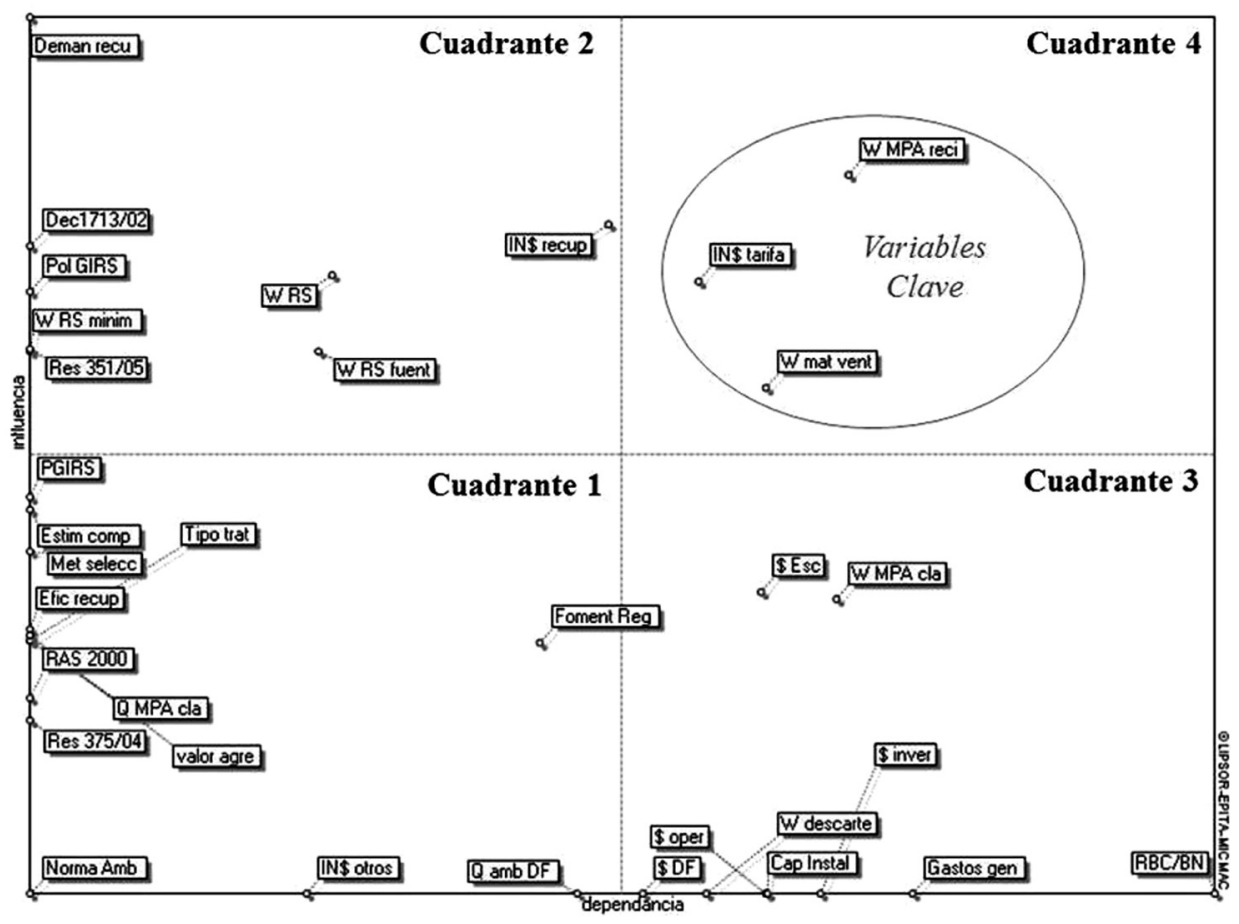

Figura 2. Plano de Influencias / Dependencias Indirectas Potenciales

la relación costo/beneficio de un proyecto PMRS, afectando su sostenibilidad a lo largo del tiempo.

\subsection{Variables clave identificadas}

En el plano de influencias/dependencias indirectas potenciales (Figura 2) se identifica la localización de variables analizadas en diferentes cuadrantes. En el cuadrante 1 se ubicaron las variables con bajo grado de influencia y de dependencia, que ejercen un impacto débil en el sistema. En este cuadrante se ubican variables como el Reglamento Técnico del Sector de Agua Potable y Saneamiento (RAS 2000) y la Resolución ICA 375 de 2004 (Res 375/04) que condicionan aspectos técnicos de las actividades de aprovechamiento y calidad de bioinsumos para actividades agrícolas, pero no están sujetas al control por parte de las administraciones de las PMRS sino que deben ser sometidas a ello. De igual forma se identifican aspectos técnicos como el método de selección (Met selecc), tipo de tratamiento de los residuos recibidos (Tipo trat) y la eficiencia de recuperación (Efic recup), los cuales si bien pueden están bajo control de las PMRS, dada su independencia y la relativa bajamedia influencia, según Godet \& Durance (2011), es preferible invertir esfuerzos variables altamente dinamizadoras como las variables clave.

En el cuadrante 2 se ubican las variables que poseen una alta influencia y baja dependencia, siendo determinadoras del funcionamiento del sistema. Ejemplo de ello son las variables políticas y normativas como la Política GIRS (PolGIRS) la Resolución CRA 351 de 2005 (Res 351/05), aspectos de mercado como la demanda de materiales recuperados (Deman recu), o sociales como la generación de residuos $(W R S)$ y de separación en la fuente por parte de la población (W RS fuent). Estas variables determinan el funcionamiento del sistema y sobre ellas la injerencia de la administración de una PMRS de es limitada.

En el cuadrante 3 se ubican las variables que tienen alta dependencia y baja influencia, lo que las constituyen como variables resultado de la dinámica del sistema analizado. Variables relacionadas con flujos de dinero como costos de inversión (\$ inver), de operación (\$ oper), gastos generales (Gastos gen), se ubican en dicho cuadrante como resultados de las interacciones de las demás variables del sistema. 
En el cuadrante 4 se ubican las variables que poseen una alta influencia y alta dependencia sobre el sistema, constituyéndose en Variables Clave. Estas variables generan cierta inestabilidad en el sistema, ya que cualquier acción que se efectúe sobre estas, propicia una serie de efectos replicadores sobre las demás variables, sobre la variable clave en sí y sobre el sistema estudiado (Godet \& Durance, 2011). Las variables clave (Figura 2) son los ingresos por tarifa de recepción de material a aprovechar (IN\$ tarifa), la cantidad de material potencialmente aprovechable a recibir ( $W M P A$ reci) y la cantidad de material recuperado para la venta ( $W$ mat venta).

La identificación del material potencialmente aprovechable a recibir (WMPA reci) como variable clave, es consecuente con las afirmaciones de Oviedo-Ocaña et al (2011) quien destaca que las variables de mayor repercusión en un sistema de aprovechamiento consisten en la calidad, cantidad y conocimiento de las características de los residuos a recibir. En cuanto a la cantidad de materiales recuperados para la venta ( $W$ mat venta), indirectamente está supeditada al mejoramiento de los métodos de selección, el tipo de tratamiento y eficiencias de recuperación, entre otros, ya que dichos aspectos como lo señala Marmolejo et al (2011) influyen sobre la efectividad de las PMRS. Por su parte los ingresos por cobro de tarifa de recepción de residuos (IN\$
Tarifa) se constituyen como una variable clave importante dado que gran parte de los ingresos de un proyecto PMRS provienen estos ingresos. Reflejo de ello son los porcentajes de ingresos reportados por SSPD (2008) donde el 41\% del total de ingresos proviene del cobro de tarifas, el $45 \%$ por aportes municipales y tan solo un $14 \%$ a partir de la comercialización de materiales.

\subsection{Escenario de simulación económica - financiera}

A partir de los resultados de la Figura 2, se puede interpretar que las interacciones dominadas por las Variables Clave condicionan el comportamiento de los costos y gastos para la normal operación de las PMRS, lo cual afecta su sostenibilidad a largo plazo. Dicha situación se refleja en el comportamiento de los indicadores de $\mathrm{BN}$ y $\mathrm{RBC}$ para un periodo de 15 años (Figura 3).

Con base en ello, el escenario de sostenibilidad propuesto plantea que con una tarifa de recepción de materiales de $\$ 17,432 / t$ (VPN año 2014), eficiencias de recuperación de biorresiduos del 33\% (SSPD, 2008; Marmolejo et al., 2009), de reciclables del 79\% (Marmolejo et al., 2009) y bajo condiciones ideales de mercado con porcentajes mínimos de venta de compost (30\%), plásticos $(96 \%)$, papeles y cartones (98\%), vidrios (99\%) y metales $(99 \%)$ (Tabla 4), se estimó que para alcanzar el punto de

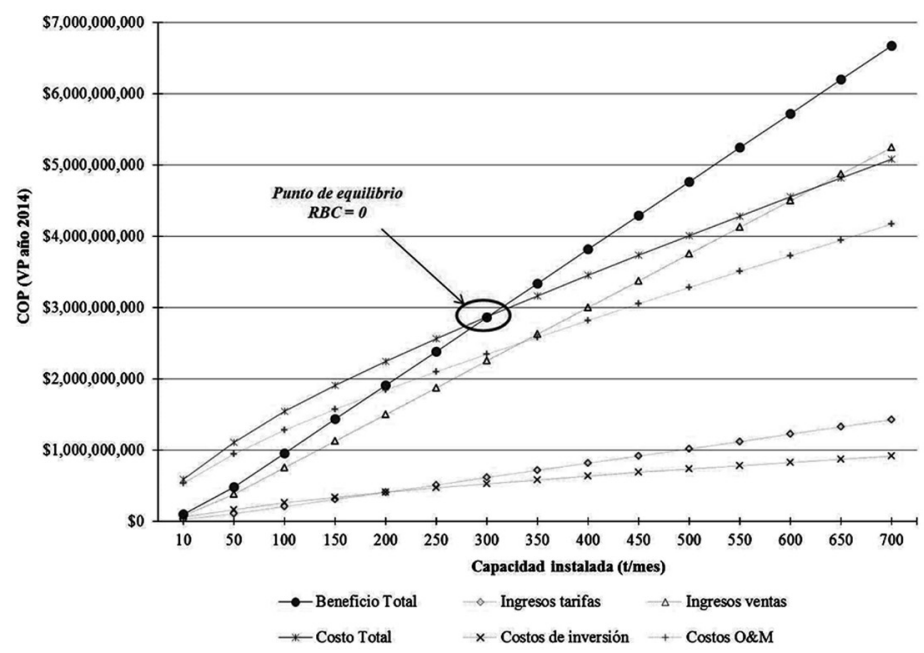

Figura 3 .Escenario de simulación de estructura de costos y beneficios acumulados - horizonte a 15 años 
Tabla 4. Constantes asumidas para simulación de costos

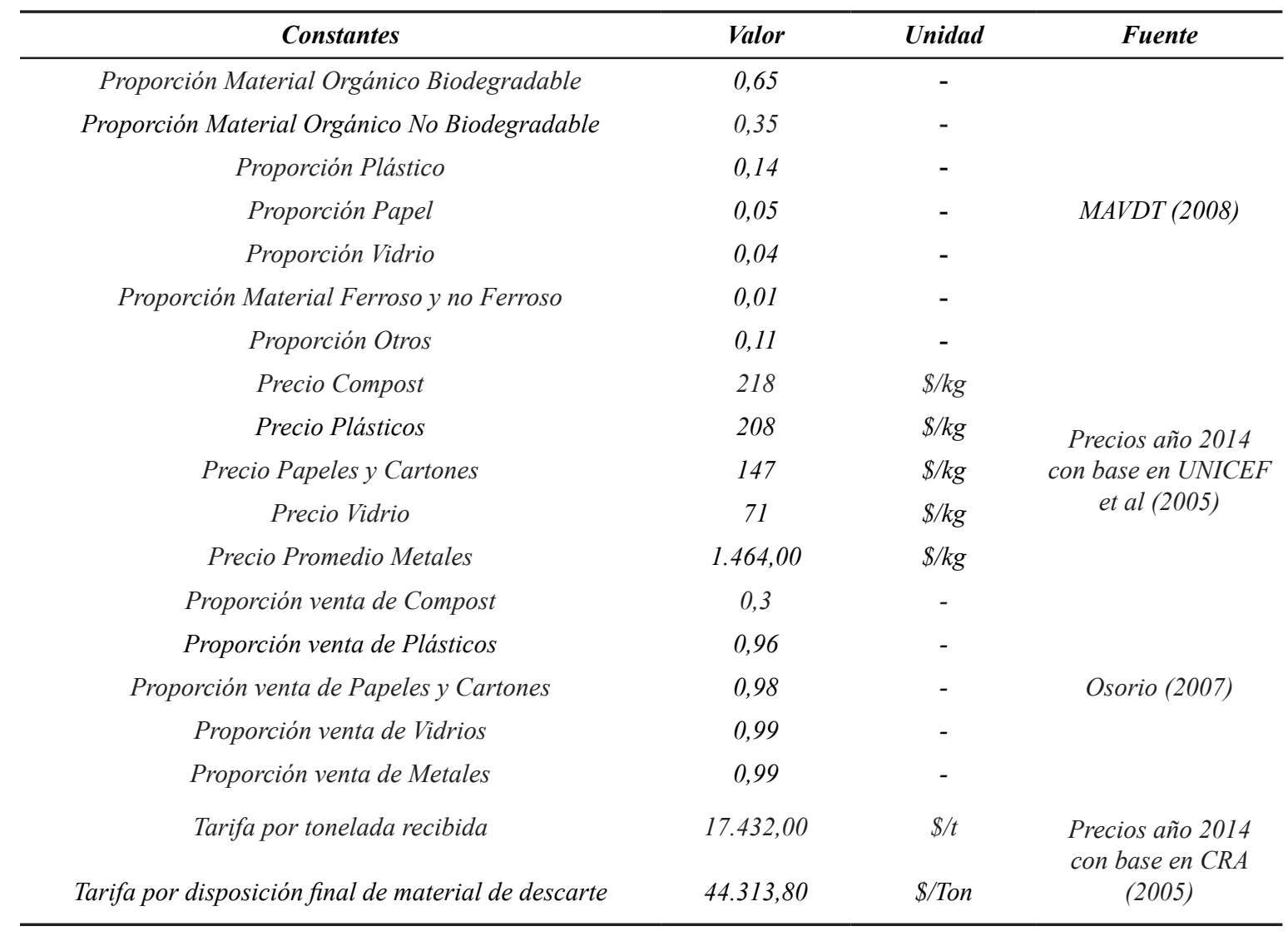

equilibrio $(\mathrm{RBC}=0$ y $\mathrm{BN}=0)$, sería necesario que una PMRS tipo recibiera como mínimo 302 t/mes, cantidad equivalente a la generación de un municipio con una población de 18,990 habitantes asumiendo una producción per cápita (PPC) de $0.53 \mathrm{~kg} / \mathrm{hab}-\mathrm{d}$ (Mindesarrollo, 2000). Bajo este escenario los ingresos ventas y recepción de residuos serían del $78 \%$ y $21 \%$ respectivamente sin necesidad de apalancar los costos con ingresos municipales, en comparación con mencionado por SSPD (2008), quien reporta valores de $32 \%$ para venta de materiales recuperados y $68 \%$ por cobro de tarifa además de subsidios municipales

Si bien, dichos resultados permiten establecer que es posible lograr la sostenibilidad económicafinanciera de una PMRS para un municipio con población inferior a 50.000 habitantes, su interpretación debe ser mesurada, ya que se basan en la existencia de unas condiciones idóneas de mercadeo de dichos materiales (Tabla 4 ), con precios de venta estables de materiales recuperados, cercanía a centros de acopio o industrias que favorezcan su comercialización. Por lo tanto se hace necesario efectuar un análisis contextual consecuente con la localización geográfica de la PMRS objeto de estudio, dado que, las condiciones mínimas para garantizar su sostenibilidad pueden variar sustancialmente, llegando inclusive a sugerir la implementación de esquemas regionalizados de recuperación/ aprovechamiento de RSUD en donde por concepto de economías de escalas se impacte positivamente las estructuras de costos.

\section{Conclusiones}

La combinación de técnicas de dinámica de sistemas (diagrama causal) y análisis estructural (matriz MICMAC), se constituye en una herramienta útil para la descripción de la dinámica entorno a la operación de una PMRS tipo en el país y para la identificación de variables clave que permitan diseñar esquemas económico- 
financieros sostenibles. Bajo los supuestos planteados, con altas eficiencias de producción de compost y recuperación/tranformación de reciclables, y con unas condiciones de mercado idóneas, para un horizonte de 15 años un proyecto PMRS, es posible alcanzar un punto de equilibrio entre ingresos y gastos totales (BN y $\mathrm{RBC}$ igual a cero) a partir del cual se pueda alcanzar la sostenibilidad de un proyecto PMRS para municipios con poblaciones menores a 50,000 habitantes. Sin embargo dicho valor puede variar en función de las condiciones regionales, tipo de tecnología a implementar, composición de los residuos de una localidad determinada entre otros, lo cual hace necesario el desarrollo de estudios concienzudos para el desarrollo de un proyecto PMRS en una localidad determinada.

\section{Referencias bibliográficas}

Abarca-Guerrero, L., Maas, G., \& Hogland, W. (2013). Solid waste management challenges for cities in developing countries. Waste Management (33), 220-232.

Bezama, A., Aguayo, P., Konrad, O., Navia, R., \& Lorber, K.E. (2007). Investigations on mechanical biological treatment of waste in South America: Towards more sustainable MSW management strategies. Waste Management 27, 228-237.

BID (Banco Interamericano de Desarrollo), AIDIS (Asociación Interamericana de Ingeniería Ambiental y Sanitaria), \& OPS (Organización Panamericana de la Salud). (2011). Informe de la Evaluación Regional del Manejo de Residuos Sólidos Urbanos en América Latina y el Caribe 2010. http://publications.iadb.org/ handle/11319/3286?locale-attribute $=$ es

Cano, C. (2011). Empleo del método MICMAC de "Análisis estructural" en la definición de factores fundamentales, para un emprendimiento efectivo en pymes de Cali-Colombia. Libre Empresa 15, 175-191.

CONPES (Consejo Nacional de Política Social y Económica) (2008). Documento CONPES 3530. Lineamientos y estrategias para fortalecer el servicio público de aseo en el marco de la gestión integral de residuos sólidos. Bogotá D.C., Colombia.

CRA (Comisión de Regulación de Agua Potable y Saneamiento Básico). (2005). Resolución 351, Bogotá D.C., Colombia

CRA (Comisión de Regulación de Agua Potable y Saneamiento Básico). (2006). Metodología de costos y tarifas para el servicio público de aseo. Bogotá D.C., Colombia.

Dong, J., Chi, J., Zou, D., Fu, C., Huang, Q., \& Ni, M. (2014). Energy-environment-economy assessment of waste management systems from a life cycle perspective: Model development and case study. Applied Energy 114, 400-408

Field, B., \& Field M. (2012). Environmental Economics: an introduction. Boston: McGrawHill Irwin.

Godet, M., \& Durance, P. (2007). Prospectiva estratégica: problemas y métodos. San Sebastián: LIPSOR - Prospektiker.

Godet, M., \& Durance, P. (2011). La prospectiva estratégica para las empresas y los territorios. Paris: UNESCO - Dunod - Fondation Prospective et Innovation.

Hoornweg, D., \& Bhada-Tata, P. (2012). What a waste: a global review of solid waste management. World Bank Urban development series, knowledge papers no. 15. Washington, D.C.

Jha, A., Singh, S., Singh, G., \& Gupta, P.(2011). Sustainable municipal solid waste management in low income group of cities: a review. Tropical Ecology 52 1, $123-131$.

Laurent, A., Clavreul, J., Bernstad, A., Bakas, I., Niero M., Gentil, E., Christensen, T.H., \& Hauschild, M.Z. (2014). Review of LCA studies of solid waste management systems - Part II: Methodological guidance for a better practice. Waste Management (34), 589-606. 
Marmolejo L. Torres, P., Oviedo, E., Bedoya, D., Amezquita, C., Klinger, R., Albán, F., \& Diaz, L. (2009). Flujo de residuos. Elemento base para la sostenibilidad del aprovechamiento de residuos sólidos municipales. Ingeniería y Competitividad 11 2, 79-93.

Marmolejo-Rebellón, L., Torres-Lozada, P., Oviedo-Ocaña, R., García, M., \& Díaz, L. (2011). Análisis del funcionamiento de plantas de manejo de residuos sólidos en el norte del Valle del Cauca. Revista EIA 16, 163-174.

Marshall, R., \& Farahbakhsh, K. (2013). Systems approaches to integrated solid waste management in developing countries. Waste Management 33, 988-1003.

MAVDT (Ministerio de Ambiente, Vivienda y Desarrollo Territorial), EPAM S.A. E.S.P. (2008). Estudios y Proyectos Ambientales y Mecánicos. Construcción de criterios técnicos para el aprovechamiento y valorización de residuos sólidos orgánicos conalta tasa de biodegradación, plásticos, vidrio, papel y cartón. Manual 1: Generalidades. Bogotá D.C, Colombia.

Ministerio de Desarrollo Económico (2000). Reglamento Técnico de Agua Potable y Saneamiento Básico - RAS. Bogotá D.C., Colombia.

Osorio, J. (2007). Modelo de aprovechamiento de residuos sólidos urbanos en Colombia. Memorias 1ra Conferencia Latinoamericana de Saneamiento (Latinosan 2007). Cali, Colombia.

Oviedo-Ocaña, E., Marmolejo-Rebellón, L., \& Torres-Lozada, P. (2011). Intervenciones priorizadas en plantas de manejo de residuos sólidos mediante la aplicación del análisis estructural. Ing. Univ. 15 1, 125-144.

PNUMA (Programa de las Naciones Unidas para el Medio Ambiente). (2013). Guía para la elaboracipón de estratégias nacionales de gestión de residuos. Nairobi, Kenia.

SSPD (Superintendencia de Servicios Públicos Domiciliarios) (2008). Diagnóstico Sectorial
Plantas de Aprovechamiento de Residuos Sólidos. Bogotá D.C., Colombia.

SSPD (Superintendencia de Servicios Públicos Domiciliarios). (2009). Situación de la Disposición Final de Residuos Sólidos en Colombia Diagnóstico 2008. Bogotá D.C., Colombia.

SSPD (Superintendencia de Servicios Públicos Domiciliarios). (2013). Situación de la Disposición Final de Residuos Sólidos en Colombia. Bogotá D.C., Colombia.

Toole, T. M. (2005). A project management causal loop diagram. In proceedings of the 21st Annual Conference of the Association of Researchers in Construction Management, London, Vol. 2, p, 763-772.

Torres, N., Olaya, C. (2010). Tackling the Mess: Causal-Loop Conceptualization of Solid Waste Management Systems through Cross-Impact Analysis. In proceedings of the 28th International Conference of the System Dynamics Society, Seoul, Korea, p. 1-13.

UNICEF (Fondo de las Naciones Unidas para la Infancia), MAVDT (Ministerio de Ambiente, Vivienda y Desarrollo Territorial), CINARA (Instituto de Investigación y Desarrollo en Agua Potable, Saneamiento Básico y Conservación del recurso Hídrico). (2005) Guía para elaborar el plan de gestión integral de residuos sólidos. Municipios menores de 50.000 habitantes. Bogotá D.C., Colombia.

Vergara, S.E., \& Tchobanoglous, G. (2012). Municipal solid waste and the environment: a global perspective. Ann. Rev. Environ. Res. 37, 277-309.

Victoria, F., Marmolejo, L., \& Torres, P. (2012). Alternativas para fortalecer la valorización de materiales reciclables en plantas de manejo de residuos sólidos en pequeños municipios. Ciencia e ingeniería neogranadina 22 (1), 59 - 73.

Zurbrügg, C., Gfrerer, M., Ashadi, H., Brenner, W., \& Küper, D. (2012). Determinants of 
sustainability in solid waste management. The Gianyar Waste Recovery Project in Indonesia.

Waste Management 32, 2126-213. 